\title{
Giuseppe DE LORENZO, Mémoires, présentation de Paola Russo, introduction de Anna Maria Rao, Vivarium, Naples, 1999
}

Renata De Lorenzo

\section{Édition électronique}

URL : https://journals.openedition.org/ahrf/1166

DOI : 10.4000/ahrf.1166

ISSN : $1952-403 X$

\section{Éditeur :}

Armand Colin, Société des études robespierristes

\section{Édition imprimée}

Date de publication : 1 décembre 2001

Pagination : 184-188

ISSN : 0003-4436

\section{Référence électronique}

Renata De Lorenzo, « Giuseppe DE LORENZO, Mémoires, présentation de Paola Russo, introduction de Anna Maria Rao, Vivarium, Naples, 1999 », Annales historiques de la Révolution française [En ligne], 326 | octobre- décembre 2001, mis en ligne le 10 mars 2008, consulté le 24 avril 2022. URL : http:// journals.openedition.org/ahrf/1166 ; DOI : https://doi.org/10.4000/ahrf.1166

Ce document a été généré automatiquement le 24 avril 2022.

Tous droits réservés 


\title{
Giuseppe DE LORENZO, Mémoires, présentation de Paola Russo, introduction de Anna Maria Rao, Vivarium, Naples, 1999
}

\author{
Renata De Lorenzo
}

\section{NOTE DE L'ÉDITEUR}

Traduction B. Gainot

1 Les commémorations du bicentenaire de la République napolitaine de 1799 ont été marquées par la publication, ou la réédition, de nombreux journaux, chroniques, récits; parmi les Mémoires, occasion d'un retour sur une participation personnelle à l'événement, mise à distance par le temps, celles de De Lorenzo (1778-1822), rédigées dix ans après les faits, dans le climat napoléonien (en 1808, l'auteur est employé au secrétariat des Finances), sont l'œuvre d'un personnage de second plan, enrôlé sans grande conviction au service de la République. Exilé en France pendant la réaction, il est revenu en Italie à la suite de Napoléon avec le grade de capitaine volontaire à la Légion italique, qui fut formée le 31 août 1799 par le Conseil des Cinq-Cents, et placée sous le commandement du général cisalpin Giuseppe Lecchi. La légion recrute dans un premier temps les jacobins exilés, en attente du retour dans leur patrie d'origine, prêts à collaborer à sa reconquête par la France.

2 Les Mémoires se terminent brusquement, lors de la formation de la République cisalpine et de la dispersion de la Légion, mais De Lorenzo est retourné à Naples en 1801 et en 1806 , sous le règne de Joseph Bonaparte. Il reprend son poste à la banque de Salvatore, ainsi que son emploi au ministère des Finances. Sans participer à la révolution de 1820-1821, il n'en est pas moins nommé en 1822, conseiller suppléant à la Cour des comptes, et il meurt la même année, âgé seulement de 44 ans. Il est donc redevenu 
comptable, sans pour autant renoncer aux valeurs politiques et militaires acquises dans les emplois napoléoniens.

3 Le récit des circonstances de son enrôlement (pp. 71-72), et surtout celui de la période de l'exil qu'il a connu dans les cités et les bourgs de France, est marqué par la séparation, et se limite à l'enregistrement des faits, sans commentaires. Le souvenir distancié, souligné par quelques remarques, je ne me souviens pas précisément (p.11), témoigne seulement avec une certaine acuité de la façon dont a été vécu ce moment dramatique, repassant dans les moments cruciaux du style du discours direct à celui du compte rendu.

4 "Narrateur candide ", susceptible de recueillir les plus infimes détails des événements de la réaction consécutive à la chute de la République, l'auteur fut considéré par Benedetto Croce, dans la première édition du manuscrit (1899) comme un homme sans idées ni initiative politique, "la bureaucratie était sa seule aspiration; il fut toujours enchanté quand il put y revenir. Homme honnête, riche de vertus privées, de celles qui ne suffisent pas à déclencher une révolution, mais quand celle-ci arrive et qu'il s'y trouve mêlé, il la sert avec zèle, fidélité et intelligence » (Giuseppe Di Lorenzo, Dans la fureur de la réaction de 1799, préface et notes de Benedetto Croce, éditions Colonnese, Naples, 1899, p. 11).

5 Benedetto Croce, aussi bien qu'Anna Maria Rao, dans leurs introductions respectives à l'une et à l'autre édition, ont insisté sur le caractère de ce républicain peu convaincu, qui fit partie de la Garde nationale, mais dont la nature profonde était celle d'un employé modèle, fidèle à sa position et dévoué à un patron quelconque, bien davantage que celle d'un militant politique attaché à un "parti». La réédition, par rapport à la première édition crocienne qui avait exclu la période qui a succédé à la réaction sanfediste à Naples, s'attache à publier intégralement le texte, dégageant tout l'intérêt de celui-ci; en fait, elle comprend la période de l'exil, et comble les omissions délibérées de Croce pour la plupart relatives à des aspects de la vie privée et de l'intimité, aux réalités quotidiennes, et aux situations de détresse. La composante sentimentale intègre la nostalgie de la patrie, mais aussi l'entourage familial, avec l'attention portée au rôle de ses parents dans son éducation, et en particulier à celui de sa mère, attentive et prévenante pour tout ce qui touche à la destinée de son fils. Figure maternelle qui marque également la formation et l'action de nombreux révolutionnaires, très différents, comme Marc-Antoine Jullien de Paris : des femmes qui élèvent dans les valeurs morales et civiques, et qui ont un grand sens pratique.

6 La publication intégrale pose en outre un problème d'analyse sémiologique et herméneutique, d'omission et de transformation des termes, qui rappelle cette oeuvre en vers bien plus connue et bien plus dense qu'est l'Essai historique sur la révolution de Naples de Vincenzo Cuoco (voir l'édition critique sous la direction d'A. De Francesco, Piero Lacaïta, Mandria-Bari-Rome, 1998.) Cela valorise le témoignage d'un auteur " mineur", en ce qu'il est susceptible de nous présenter la perception ordinaire des événements, qui fut celle de la majeure partie des contemporains.

7 La vie quotidienne dans l'exil, dimension neuve, faite de souffrance et de désillusion, mais aussi d'espérance ranimée, recouvre une gamme très étendue de situations. Cette expérience apparaît par conséquent différente de celle, bien plus engagée, d'un Cuoco, déja cité, d'un Francesco Lomonaco, et de tant d'autres parmi ceux qui ont retenu l'attention de A. M. Rao (Les exilés. L'émigration politique italienne en France [1792-1802], Guida, Naples, 1992), mêlée ensuite à l'espoir déçu de la construction d'une nation 
italienne. A une historiographie attentive au débat idéologique, va donc s'ajouter ce récit du désengagement, lequel est tout aussi essentiel pour comprendre l'atmosphère générale, un monde qui cherche à se faire une opinion. En fait, à travers ce désengagement, se révèle un aspect de la politique, se retrouve un milieu pour lequel l'expérience subjective, à commencer par celle du Triennio de 1796-1799, est une façon de trouver ses marques pour assumer les moyens de sa propre existence et de sa survie.

En ce sens, les Mémoires sont une biographie collective. L'auteur est emblématique de la présence non marginale $\mathrm{du}$ XVIII ${ }^{\mathrm{e}}$ siècle napolitain, des personnages éduqués à la valorisation des talents et des compétences comme modes de promotion sociale (en l'espèce, les capacités en matière de comptabilité administrative), mais dans le cadre de la stabilité de l'Ancien Régime. Catholiques par tradition familiale, passés par les écoles et les maîtres traditionnels, ils se sont rapidement adaptés à l'éducation politique, à la lutte, à l'exil. E. De Lorenzo est ainsi l'individu-groupe marqué par des expériences successives.

9 La «médiocrité » du personnage n'exclut pas sa participation aux mouvements d'incertitude, de désordre, qui se concentrent, pour conserver une tonalité générale et une cohérence narrative, sur: 1 - La République napolitaine, la répression vécue à travers la fuite dans les rues de la capitale, 2 - La prison, 3 - L'exil. Ce qui correspond à divers espaces physiques et mentaux, et se rapporte à diverses formes de sociabilité.

La République est un moment historique qui n'a pas réussi à atteindre sa normalité propre et ses objectifs de réformisme constructif; la cité n'est pas constituée comme organisation sociale (p. XXV) et administrative. Tout cela se passe dans l'urgence, dans le vécu immédiat et dans la mémoire, il subsiste surtout la violence, les moments d'« anarchie ", par lesquels s'ouvrent les premières journées, et dans laquelle sombrent les journées finales des 13 juin et suivantes. À propos de cette issue, A. M. Rao souligne chez De Lorenzo le brio avec lequel celui-ci réussit à peindre le réseau urbain, les espaces parcourus maintes fois dans la recherche désespérée des secours (p. XXII), privilégiant dans cette recherche la protection des parents, les lieux professionnels, ceux des récentes fonctions militaires, de la pratique religieuse, du pouvoir. Les rues, espaces de la fuite, qui délimitent l'espace citadin de la réaction (p. XXIV), comme dans de nombreuses autres chroniques (Caria De Nicola, Bartolomeo Nardini, Diomede Malinelli, Stefano Pistoia, Emmanuele Palermo), où la motivation de base est l'exigence d'ordre et de restauration des bons, que l'on souhaitait en janvier, et qui a fait place aux jours de terreur (p. 11), lors de l'entrée de Championnet à Naples.

11 La représentation du peuple est faite en réalité d'anarchie et de désordre. La révolution propulse De Lorenzo de la place de comptable à celle de militaire, tout d'abord en exerçant le même emploi dans la compagnie du capitaine Giuseppe Serra, duc de Cassano, puis celui de secrétaire de la deuxième légion de la Garde nationale, pour se tenir ensuite à l'écart de celle-ci, et enfin de nouveau dans la sixième compagnie du deuxième bataillon comme capitaine. Les événements républicains sont tout entier appréhendés du point de vue du sujet narrateur, et ramenés à quelques moments de sa vie : la mort de la mère, la garde civique, la nomination dans la légion. Les événements politiques sont occultés ou placés à l'arrière-plan, les jugements allusifs (les actions inopportunes du gouvernement napolitain devant l'avance de Ruffo sont qualifiées de bévues, p. 191. La République apparait sur le devant de la scène à travers l'auteur, et à travers nombre de protagonistes héroïques, mais mineurs, dans les phases tragicohéroïques du désordre et de la chute. C'est au cours de cette dernière période que 
l'auteur perçoit le peuple napolitain comme de la canaille hautement scélérate (p. 52, expression supprimée par Croce), unique, incomparable à n'importe quelle nation rencontrée dans l'exil.

12 La prison est un lieu de rassemblement propice pour commettre de nouveaux abus de pouvoir et des spoliations. De Santa Maria Apparente aux Granili, celles-ci sont placées sous l'autorité de chefs de poste militaire, de commandants pillards et sanguinaires, souvent des criminels notoires, qui y trouvent l'occasion de s'enrichir, au détriment des individus et des familles. Là, l'individu se transforme, prêt à devenir fourbe, à inventer un système pour survivre, là se nouent des amitiés nouvelles, et s'opérent de faux rapprochements éphémères, là il est possible de faire carrière.

13 La nouvelle, après six mois de prison, de la condamnation à dix années d'exil, n'est pas un moment négatif. Elle met un terme à la douleur de l'existence violente et accablante de la prison, et surtout elle ouvre l'interrogation, partagée par de nombreux républicains, moins irrésolus, plus convaincus, sur le moment auquel le retour deviendra possible, en fonction du changement de la situation internationale, et de la lutte pour influer sur celle-ci. L'angoisse, la vie au jour le jour, la souffrance, font place à l'espoir retrouvé.

14 L'exil, commencé le 12 décembre 1799, signifie un voyage périlleux et un rapport avec la France entamé sous un angle différent de celui de l'époque républicaine. La partie relatée de cet exil, et pas seulement le voyage de départ, omis par Croce, est au contraire le prétexte à des rencontres, à des petites anecdotes individuelles qui mettent en scène les grands protagonistes, nonobstant le désengagement politique de l'auteur, loin des disputes qui s'élèvent entre ses compatriotes. C'est l'occasion en réalité de rencontrer Napoléon, présence charismatique et quasi miraculeuse, en trois occasions ; et c'est là comme le souligne A. M. Rao (p. XXIII) l'un des rares passages où la " grande " histoire croise la vie quotidienne. De Lorenzo eut cette occasion à Toulon, où le bateau arrive le $1^{\text {er }}$ janvier 1800 , au cours d'une manifestation pour célébrer la nouvelle constitution de l'an VIII donnée à la République par le général promu depuis peu Consul de la République française. Puis à Lausanne, où il l'a rencontré en personne, et enfin lors du passage du Grand-Saint-Bernard.

Il effectue un voyage entre tourisme et diaspora, commencé avec l'exil, lorsqu'il se retrouve dans une situation de touriste par hasard et par nécessité (p. XXXVI), avec de fréquents déplacements à pied et à marche forcée, ce qui n'empêche pas des remarques sur les monuments et les œuvres d'art. De Toulon à Beausset, à Aubagne et à Marseille, à Dijon, pour finir en Suisse, à Genève, Lausanne, à Milan, Lecco, Brescia, Pizzighettone, Pavie, Varese. Lieux dans lesquels il ressent un intense découragement et une dépression, mais aussi l'expérience vécue dans les premiers jours d'avril du passage du Grand-Saint-Bernard, avec l'armée de 80000 hommes, entreprise pionnière, racontée avec la conscience de vivre une expérience unique.

Les citoyens contraints par les municipalités d'offrir l'hospitalité aux exilés présentent un tableau varié d'individualités et de familles. Le problème récurent du logement, la plupart du temps dans des domiciles privés, permet d'avoir des rapports avec la population, de sonder les humeurs, de vivre aussi des expériences étrangères à son statut personnel. Au cours de ces rencontres, la consolation vient bien souvent des gens simples, davantage que des politiciens. L'encasernement militaire, plus moderne, génère par ailleurs de nouvelles formes de discrimination et de privilèges entre officiers. 
17 Le service volontaire dans l'année d'Italie traduit le passage du soldat bureaucrate au service militaire actif; pour la première fois, l'auteur goûte les fatigues, les risques et les soucis de la guerre, c'est-à-dire les marches, les repos à ciel ouvert, la nourriture pas toujours suffisante, l'abri précaire. C est aussi la voie de l'éducation du soldat-citoyen, ainsi que l'occasion de rencontre avec d'autres exilés italiens, une façon de dépasser l'étroite vision municipaliste pour une appréhension italienne du problème politique. Dans l'armée napoléonienne, De Lorenzo pratique une façon d'être militaire différente de celle qu'il a expérimentée durant la République, quand un compromis avec son ancien statut de comptable était encore possible : la participation à la première bataille après le passage du Grand-Saint-Bernard, au cours de laquelle se distinguèrent nombre d'officiers napolitains, le combat en duel avec un Français, l'attaque des villes, la capture de prisonniers, le risque d'être tué. De la même façon, on imagine les conflits internes à la Légion entre les militaires professionnels et les sous-officiers volontaires, ces contradictions rendues familières par les Mémoires des militaires, qui renvoient à une contradiction interne à l'armée, nourrie des motifs les plus variés, depuis l'origine nationale jusqu'aux rancœurs, aux modes de vie différents et aux insultes. C'est à ce nouveau type d'armée de l'État napoléonien, tout autant respectueux des règles de la confrontation militaire, en situation précaire, qu'ouvert aux talents, que va finalement se convertir le comptable De Lorenzo.

18 La vie militaire, entreprise et poursuivie de mauvais gré, sans élans héroïques, mais avec la promesse et la volonté de participer aux principes de liberté et de démocratie découverts avec la révolution, représente essentiellement un saut qualitatif par rapport à la cruauté des premières pages, à la situation d'errance sur les places et dans les ruelles pour échapper aux sanfédistes. Au terme de cette vie militaire, se fait jour le désir de retourner à un travail plus humble, marque d'une double réaction à la militarisation forcée, à la fois dans la recherche de nouvelles opportunités, mais aussi par un désir de stabilité qui clôt l'expérience guerrière.

19 Avec la victoire de Marengo et l'armistice, les 2500 hommes de la Légion italique reçurent leur solde, et furent mis au service de la République cisalpine; à Milan, le gouvernement cisalpin provoqua la désillusion, avec l'encasernement dans des locaux insalubres, situation à laquelle on tentait d'échapper en préférant les jardins publics, avec les promesses non tenues d'employer les légionnaires dans les nouveaux régiments, avec le regain de la défiance et de l'hostilité politique à l'égard des méridionaux qui retournent en Italie après l'exil en France. L'exclusion du service dans le nouveau corps de bataillon des officiers italiens, qui condamnait à une situation financière précaire au sein de la Cisalpine, fut vécue comme une opération de marginalisation, une ingratitude que justifia le général Masséna en invoquant une mesure d'économie prise par la république naissante.

Dans ce nouveau monde, précaire et instable, les amis, les collègues, furent sans doute irrités, mais nul ne contesta les mesures de persécution. À cela contribuent également les partis pris du récit lui-même qui, par exemple, ne fait pas référence aux nombreux officiers napolitains rassemblés à Pavie, et la mentalité d'un militaire essentiellement préoccupé par les difficultés immédiates, par les données logistiques, et en priorité par la qualité du logement.

21 Pour terminer, les Mémoires se présentent comme le récit du désenchantement qui imprégna l'esprit de ceux qui ne s'engagèrent pas dans le mouvement démocratiquepatriotique avec une perspective de relance de la lutte politique en Italie, de ceux qui 
vécurent l'exil comme une perpétuelle errance, à la recherche de certitudes, géographiques, physiques, et existentielles. Le choc de la rencontre avec la réalité française fut également pour eux une approche personnalisée de la politique, l'ouverture sur un monde non monolithique, aux réalités multiples, idéalisé dans l'esprit de ceux qui ont combattu au nom des idéaux républicains, essentiel à l'acquisition de paramètres en vertu desquels il devient possible d'œuvrer dans l'avenir.

22 L'intention punitive qui fut à l'origine de l'émigration des républicains italiens s'est transformée en une œuvre constructive de modification des idées et des mentalités. La communauté dans laquelle se reconnaissent progressivement des hommes tels que le comptable De Lorenzo sera l'État éclairé de l'ère napoléonienne. 